\title{
Hyperbaric oxygen therapy improves colorectal anastomotic healing
}

\author{
G. S. A. Boersema ${ }^{1} \cdot$ Z. Wu ${ }^{1,2} \cdot$ L. F. Kroese ${ }^{1} \cdot$ S. Vennix ${ }^{1,3}$. \\ Y. M. Bastiaansen-Jenniskens ${ }^{4}$ - J. W. van Neck ${ }^{5}$ - K. H. Lam ${ }^{6}$ • G. J. Kleinrensink ${ }^{7}$ • \\ J. Jeekel ${ }^{7}$ J. F. Lange ${ }^{1}$
}

Accepted: 22 March 2016/Published online: 4 April 2016

(C) The Author(s) 2016. This article is published with open access at Springerlink.com

\begin{abstract}
Purpose Hyperbaric oxygen treatment (HBOT) has been found to improve the healing of poorly oxygenated tissues. This study aimed to investigate the influence of HBOT on the healing in ischemic colorectal anastomosis.

Methods Forty Wistar rats were randomly divided into a treatment group that received HBOT for 10 consecutive days ( 7 days before and 3 days after surgery), or in a control group, which did not receive the therapy. Colectomy with an ischemic anastomosis was performed in all rats. In each group, the
\end{abstract}

G. S. A. Boersema and Z. Wu contributed equally to this work.

Electronic supplementary material The online version of this article (doi:10.1007/s00384-016-2573-y) contains supplementary material, which is available to authorized users.

Z. Wu

wuzhouqiao@gmail.com

1 Department of Surgery, Laboratory of Experimental Surgery, Erasmus MC, University Medical Center, Room Ee-173 Postbus 2040, 3000 CA Rotterdam, The Netherlands

2 Department of Gastrointestinal Surgery, Peking University Cancer Hospital and Institute, Beijing, China

3 Department of Surgery, Amsterdam Medical Center, Amsterdam, The Netherlands

4 Department of Orthopaedics, Erasmus MC, University Medical Center, Rotterdam, The Netherlands

5 Department of Plastic and Reconstructive Surgery, Erasmus MC, University Medical Center, Rotterdam, The Netherlands

6 Department of Pathology, Erasmus MC, University Medical Center, Rotterdam, The Netherlands

7 Department of Neuroscience, Erasmus University Medical Center, Rotterdam, The Netherlands rats were followed for 3 or 7 days after surgery to determine the influence of HBOT on anastomotic healing.

Results Five rats from each group died during follow-up. No anastomotic dehiscence was seen in the HBOT group, compared to $37.5 \%$ and $28.6 \%$ dehiscence in the control group on postoperative day (POD) 3 and 7, respectively. The HBOT group had a significantly higher bursting pressure (130.9 $\pm 17.0 \mathrm{mmHg})$ than the control group $(88.4 \pm 46.7 \mathrm{mmHg}$; $\mathrm{p}=0.03$ ) on POD 3. On POD 3 and POD 7, the adhesion severity was significantly higher in the control groups than in the HBOT groups $(p<0.005)$. Kidney function (creatinine level) of the HBOT group was significantly better than of the control group on POD $7(p=0.001)$. Interestingly, a significantly higher number of CD206+ cells (marker for type 2 macrophages) was observed in the HBOT group at the anastomotic area on POD 3.

Conclusion Hyperbaric oxygen enhanced the healing of ischemic anastomoses in rats and improved the postoperative kidney function.

Keywords Anastomotic healing · Hyperbaric oxygen therapy $\cdot$ Animal model $\cdot$ Perfusion

\section{Introduction}

Colorectal anastomotic leakage (CAL) is the most serious complication following colorectal surgery, causing substantial morbidity and mortality as high as $33 \%$ [1]. With continuous improvements in surgical techniques and perioperative care, the incidence of this complication still varies between 10 and $13 \%$ [2, 3], hardly decreasing in recent decades despite developments in medical science and technology [4]. 
Regional ischemia has been considered as one of the main causes of anastomotic leakage [5-9]. Poor blood supply and perfusion of the rectal stump and ascending loop of bowel, or prolonged hypoxia are detrimental for wound healing and thus increase the risk of CAL $[5,10,11]$. Poor perfusion delays wound healing processes $[12,13]$. The blood supply and perfusion of the preserved bowel, especially at the cutting edge, significantly affects the outcome of patients. Injury to the colon following an ischemic event is due to hypoxia and to reperfusion injury. Bowel ischemia results in hypoxia of the cells. Within $1 \mathrm{~h}$ of ischemia, injury in the superficial part of the mucosa is already detectable. Prolonged severe ischemia causes necrosis of the mucosa layer, and lead to transmural infarc-

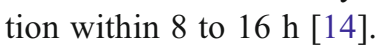

To prevent ischemia, one direct intervention is to provide oxygen. Oxygen is an essential component in tissue repair and wound healing. Oxygen stimulates collagen synthesis, matrix deposition, angiogenesis, epithelialization, and the eradication of bacteria [15-17]. Perioperative use of oxygen has been reported to reduce CAL and improve patient's outcome after colorectal surgery [18]. Clinical data have shown that perioperative hyperoxygenation reduced the occurrence of surgical site infections [19]. However, consensus in the interpretation of the data in this regard has not yet been reached and the mechanism of the oxygen therapy is still yet to be established.

The use of hyperbaric oxygen therapy (HBOT) is based on the same principle of hyperoxygenation and has been introduced in the treatment of surgical patients as well as in the treatment of patients with chronic wounds $[20,21]$. A positive influence of HBOT on anastomotic healing was first reported by Hamzaoglu et al. in 1998 [22]. Although this therapy is widely used in medical practice, its mechanism of action is still poorly understood. Previous study from Attard et al. has suggested that application of HBOT may reduce the production of inducible nitric oxide synthase protein (iNOS) expression [23], which is actively involved during the occurrence of CAL [24]. Many previous studies focused on localized changes such as collagen deposition and MMP (matrix metallopeptidase) activities [25].

The accumulated data supports the hypothesis that HBOT may improve anastomotic wound healing via suppression of pro-inflammatory agents and stimulation of anti-inflammatory agents [26]. This study was carried out to verify this hypothesis in an experimental rat model. HBOT was applied daily to all the rats 7 days prior to a partial colectomy with ischemic anastomosis, until 3 days after surgery. Macroscopic evaluation of anastomotic healing evaluation and immunohistochemistry were performed to investigate involvement of different inflammatory agents.

\section{Materials and methods}

\section{Animals}

Forty male Wistar albino conventional rats, $300-350 \mathrm{~g}$, were purchased from a licensed breeder (Harlan Laboratories, Boxmeer, The Netherlands). All rats were bred under specific pathogen-free conditions and kept under standard laboratory conditions in individually ventilated cages, and had ad libitum excess to water and regular rat chow. The experimental protocol was approved by the local Ethical Committee of Animal Experimentation of Erasmus University Rotterdam.

\section{Experimental design}

The animals were randomly divided into four different groups: control group, 3 days follow-up; control group, 7 days followup; HBOT group, 3 days follow up; HBOT group, 7 days follow-up.

The HBOT groups received HBOT for 10 consecutive days from 7 days prior to surgery until 3 days after surgery. Each HBO (hyperbaric oxygen) session consisted of $100 \%$ oxygen under a pressure of $2.4 \mathrm{~atm}$ absolute for $90 \mathrm{~min}$ in the HBO Test Vessel P1460 [27]. Animals were placed in a large transportation box (ten animals together) during the session. To exclude the possible bias due the HBOT procedure itself rather than the therapy itself, the control groups were also placed in a transportation box for the same time period in the same room, however, without undergoing HBOT.

On the day of operation, rats were anesthetized using $2 \%$ isoflurane/O2; in addition, preoperatively, $0.05 \mathrm{mg} / \mathrm{kg}$ buprenorphine was administered as pain medication. After shaving and disinfecting the abdomen, the abdominal wall was opened through a 5-cm laparotomy. Subsequently, the ileocecocolic arteries, the right colic artery, the middle colic artery, and the left colic artery were ligated (Silkam 4/0, B. Braun, Germany) to create an ischemic anastomosis (Fig. 1). A partial colectomy was performed and proximal and distal ends of the colon were invertedly anastomosed with Dafilon 8/0 (B. Braun, Germany). This model is earlier described, but in short, the colonic segment between $1.0 \mathrm{~cm}$ aborally to the cecum and $0.5 \mathrm{~cm}$ above the caudal mesenteric artery was resected [28]. The end-to-end anastomosis in all groups was made with 12 continuous sutures. Finally, the abdomen was closed in two layers and the rats were resuscitated with $5 \mathrm{~mL}$ of normal saline solution subcutaneously to prevent dehydration. No antibiotics were used. The HBO groups received HBOT immediately after surgery, which continued on postoperative day (POD) 1 and POD 2, and on POD 3 just before the reoperation. During follow-up the rats were weighted and observed daily and had ad libitum access to water and food. 


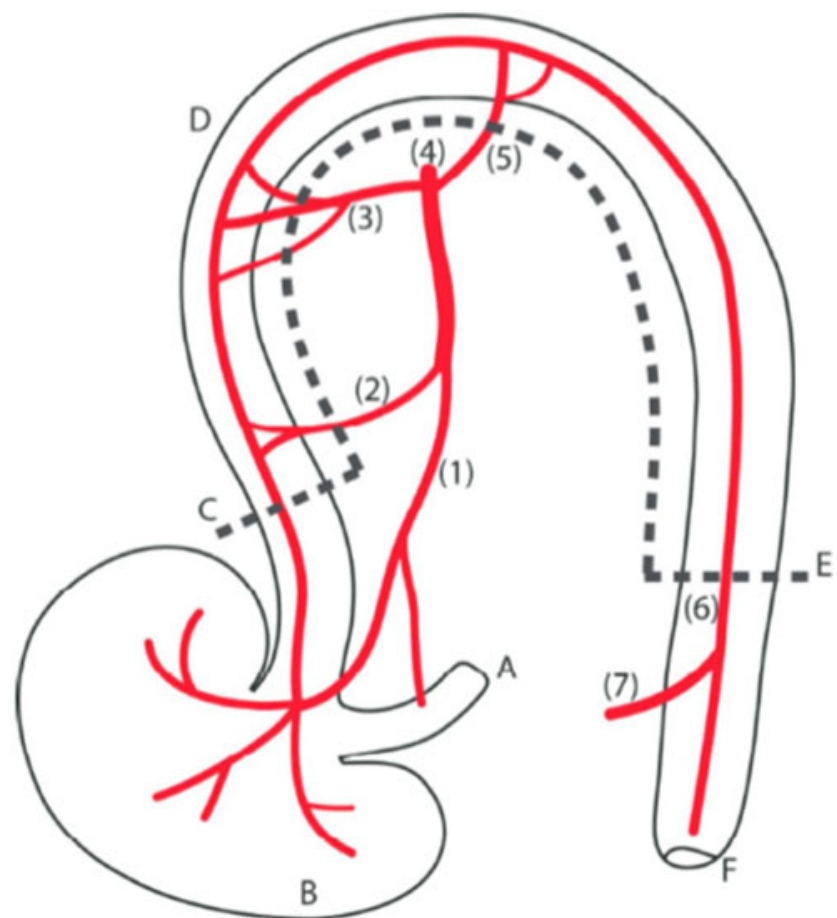

Fig. 1 Schematic overview of artery distribution in rat colon, anterior view. (1) ileocecocolic artery, (2) colic branch of ileocecocolic artery, (3) right colic artery, (4) cranial mesenteric artery, (5) middle colic artery, ( 6 ) left colic artery, (7) caudal mesenteric artery, $(A)$ terminal ileum, $(B)$ cecum, $(C)$ proximal cutting edge, $(D)$ colon, $(E)$ distal cutting edge, $(F)$ anus. Adapted from $\mathrm{Wu}$ et al. [28]

\section{Assessment of the anastomoses}

\section{Clinical observation and physical examination}

On POD 3 or POD 7, rats were anesthetized again. Anastomotic healing was assessed by observational, physical, and histological examination. The abdomen was checked for signs of anastomotic dehiscence. Abscess formation was scored according to the following scoring system: $0=$ no abscess; $0.5=$ one very small abscess; $1=$ several small abscesses; 2 = medium abscess; 3 = large or several medium abscesses; $4=$ one very large or several large abscesses [29, 30]. Adhesion strength and amount were recorded using the Zühlke score [31]. After clinical observation, the anastomotic bursting pressure test (ABP) was recorded in the same way as described previously [28]. In short, the ABP was determined by insufflation of air in the closed segment of the colon, and the first leak of air was noted as the bursting pressure, the location was also noted.

\section{Serum measurement}

On POD 3 or POD 7, creatinine levels were measured in the serum using the QuantiChrom assay kits (DIUR-500 and DICt-500, Gentaur Europe, Brussels, Belgium).
Histopathological evaluation

After the measurement of bursting pressure, a 1-cm-long colonic segment, $0.5 \mathrm{~cm}$ on each side of the anastomosic line, was resected and prepared for histopathological examination using standard procedures [28]. HE staining was performed and the anastomotic area of the slides was scored using the Ehrlich and Hunt numerical scale as modified by Phillips et al. [32]. The scoring system evaluated four parameters: inflammatory cell infiltration, fibroblast activity, development of new blood vessels, and collagen deposition. The parameters were graded from 0 to 4 as follows: $0=$ no evidence, $1=$ occasional evidence, 2 =light scattering, $3=$ abundant evidence, $4=$ confluent cells or fibers. The samples were scored by three investigators (G.B, Z.W. K.L.), who were blinded for the clinical findings, group allocation, and the treatment. Immunohistochemical staining for iNOS (marker for macrophage type 1; M1, 1:400, Abcam plc, Cambridge, UK, secondary antibody rabbit-anti-rabbit) and CD206 (marker for macrophage type 2; M2, 1:1600, Abcam, secondary antibody rabbit-anti-rabbit) were also performed on anastomotic samples with the same method described previously [24]. After an overnight incubation at $4{ }^{\circ} \mathrm{C}$, the slides were incubated with Envision secondary antibody (DAKO, Glostrup, Denmark). After $30 \mathrm{~min}$, diaminobenzidine (DAKO, Glostrup, Denmark) was used for visualization of antigen-antibody reactivity. Slides were counterstained with hematoxylin.

To determine the positive target cell number on each slide, the same five fields were selected at the anastomotic site on each slide using a microscope with an imaging system (Olympus DP25, Tokyo, Japan), under 20× 10 magnification $(2560 \times 1960$ pixels $)$. The cell numbers were counted with ImageJ (National Institutes of Health, Bethesda, MD). The average cell number of the selected fields was used for analysis. An M2/M1 index was calculated with the following equitation. The natural logarithm was used to adjust the data to normal distribution.

$\mathrm{M} 2 / \mathrm{M} 1$ index $=\ln \frac{\text { Number of } \mathrm{CD} 206+\text { cells }}{\text { Number of iNOS }+ \text { cells }}$

\section{Statistical analysis}

Statistical analysis was performed with SPSS 21.0 (IBM Inc., Chicago, USA). Data are presented as mean \pm standard deviation (S.D.) or as median or as percentage. The Mann-Whitney $U$ test, $t$ test, and Pearson's correlation test were used according to proper indications. We used the Levene's test to test equality of variances. The one-way analysis of variance was performed with the Kruskall-Wallis test for non-parametric parameters. All reported $p$ values were two-sided; a $p$ value $<0.05$ was considered to indicate statistical significance. 
Table 1 Comparison of anastomotic leakage and colon anastomoses abscess rate on POD 3 and POD 7 in the control and HBO groups

\begin{tabular}{|c|c|c|c|c|c|}
\hline & \multicolumn{2}{|l|}{ POD 3} & \multicolumn{2}{|l|}{ POD 7} & \multirow[t]{2}{*}{$p$ value } \\
\hline & control & $\mathrm{HBO}$ & control & $\mathrm{HBO}$ & \\
\hline Number of rats & 10 & 10 & 10 & 10 & \\
\hline Mortality (\%) & $20(2 / 10)$ & $20(2 / 10)$ & $30(3 / 10)$ & $30(3 / 10)$ & NS \\
\hline Anastomotic dehiscence $(\%)$ & 37.5 & 0 & 28.6 & 0 & 0.02 \\
\hline \multicolumn{6}{|l|}{ Colon anastomoses } \\
\hline Abscess (mean number) & 4 & 2 & 2 & 0 & 0.08 \\
\hline
\end{tabular}

Dehiscence and abscess rate only include surviving animals

\section{Results}

\section{Overall and general observation}

In both the control groups and HBOT groups 5 animals died; all deaths are unrelated to anastomotic leakage: 5 colon ischemia, 4 colon ischemic necrosis, and 1 overdosed anesthesia. Postoperative weight loss occurred in all rats without significant difference between the groups.

\section{HBOT improves clinical parameters}

Anastomotic dehiscence was strictly limited to the control groups. The POD 3 group had $37.5 \%(3 / 8)$ leakage and the POD7 group $28.6 \%(2 / 7)$ versus a rate of $0 \%$ in both HBOT groups $(p=0.021)$. The number of abscesses between groups was not significantly higher in the control groups $(p=0.08)$ (Table 1). HBOT resulted in significantly less anastomotic adhesions, which were significantly less severe as without oxygen therapy on both POD 3 and POD 7 evaluated with the Zühlke score (Fig. 2).

On POD 3, the anastomotic bursting pressure (ABP) was significantly higher in the HBOT group than in the control group: $130.9 \pm 17.0 \mathrm{mmHg}$ vs. $88.4 \pm 46.7 \mathrm{mmHg}(p=0.03)$ (Fig. 3). The variance of ABP in POD 3 in the HBOT group was also significantly lower $(p=0.004)$. ABP was not significantly different between the HBOT group and control group at POD $7162.4 \pm 39.7 \mathrm{mmHg}$ vs. $141.1 \pm 73.3 \mathrm{mmHg}$ $(p=0.51)$, but the variance of ABP was significantly lower in the HBO group $(p=0.009)$.

The mean creatinine level in the HBOT group on POD 3 was lower, though not significantly, than in the control group; $13.4 \pm 9.0$ vs. $30.3 \pm 28.6 \mathrm{mg} / \mathrm{dl}(p=0.07)$. On POD 7 , the creatinine levels were significantly lower in the HBOT group than in the control group, $9.0 \pm 12.1$ vs. $52.0 \pm 25.2 \mathrm{mg} / \mathrm{dl}$ (Fig. 4).

\section{Histology evaluation}

The inflammatory cell infiltration, fibroblast activity, neoangiogenesis, and collagen deposition based on the HE staining were not evidently different between the groups (Supplementary data 1). After 7 days more angiogenesis was observed in the HBOT group. Based on immunochemistry, significantly more CD206+ cells (M2) were present in the HBOT group than in the control group on POD $3 ; 50.0$ \pm 28.1 vs. $19.4 \pm 16.2$ cells, $p=0.016$ (Fig. 5). Also the M2/ M1 index was significantly higher in the HBOT short-term group; $p=0.02$. The number of iNOS+ cells as a marker for type 1 macrophages (M1, pro-inflammatory marker) was higher in the short-term control group although not significantly different (Fig. 5).
Fig. 2 Adhesion score (number of adhesions) and Zühlke score (strength of adhesions) around the anastomosis [31]. The adhesions on POD 7 in the HBOT were significantly less firm; control 3.7 \pm 1.1 versus HBOT $1.29 \pm 0.8$. $*$ indicate significance $(p=0.001)$
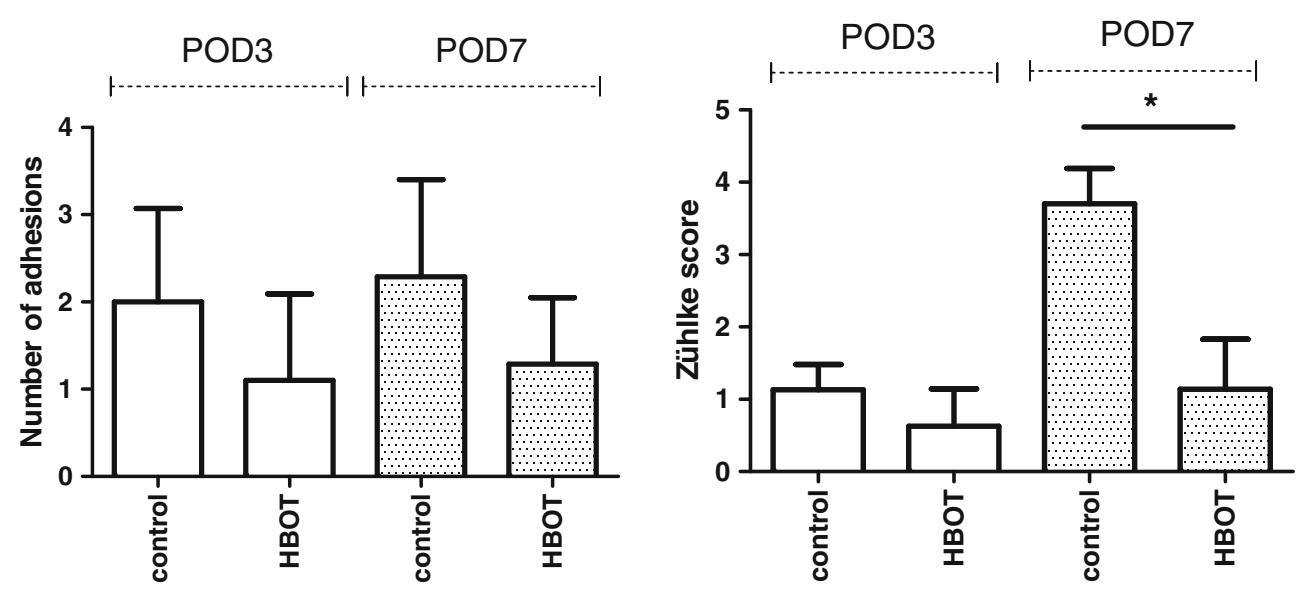


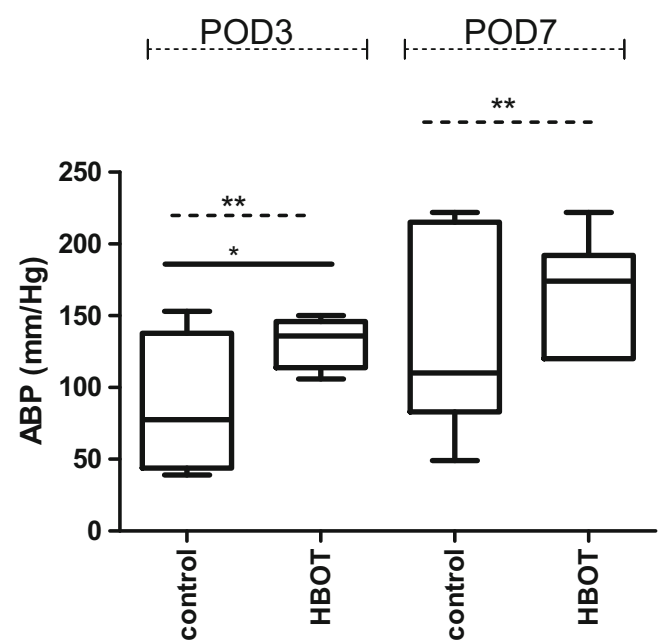

Fig. 3 The anastomotic bursting pressure (ABP) in $\mathrm{mmHg}$, was significantly higher on POD3 in the HBOT group $130.9 \pm 17.0 \mathrm{mmHg}$ vs. $88.4 \pm 46.7 \mathrm{mmHg}, \mathrm{p}=0.03$. On POD7 we found a trend to an higher ABP in the HBOT group, but not significant $(p=0.098)$, although the variance was significantly lower in the HBOT POD7 group. The variance of ABP between the groups was significantly different and is indicated with the $p$ value on the dash line. *indicate $p<0.05$, * indicate $p<0.01$

\section{Discussion}

Colorectal anastomotic leakage is a dangerous short-term complication after colorectal surgery and may cause substantial immediate mortality if not treated as soon as possible. In this study, we evaluated the influence of HBOT on ischemic anastomotic wound healing and we found that HBOT improved the wound healing in ischemic colorectal anastomosis as shown with a higher ABP and less firm adhesions. We also observed improved postoperative recovery based on higher creatinine levels in the rats that received HBOT.

When comparing the data from this ischemic colorectal anastomosis model to the standard rat colectomy model [28] and to the other ischemic anastomosis models, the ligation of the arteries of the ascending stump resulted in catastrophic outcomes including evident ischemia at the cecum after surgery and a mortality rate as high as $25 \%$. This is comparable to the clinical situation in ischemic bowel patients [33]. To our knowledge, this model best mimics clinical outcomes also because most ischemic anastomosis models only cause a lower bursting pressure and localized changes after surgery. Our previous study showed that functional failure of the ascending stump perfusion results in CAL in patients [34]. Impaired tissue perfusion may result from patient-related factors such as smoking inflammatory bowel disease or diabetes [35-37]. Meanwhile, different technique-related factors such as the level of artery ligation and anastomosis configuration may also influence anastomotic perfusion [34]. Our model inflicted a severe ischemic injury to the standard

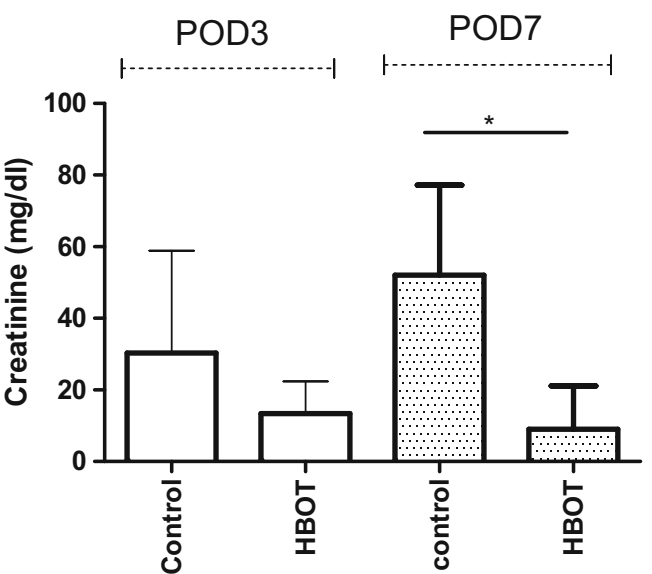

Fig. 4 Creatinine levels measured as $\mathrm{mg} / \mathrm{dl}$ were significantly higher in the control group than in the HBOT group on POD7; $9.0 \pm 12.1$ vs. 52.0 $\pm 25.2 \mathrm{mg} / \mathrm{dl}, p=0.003(*)$

colorectal anastomosis, providing a satisfactory environment for evaluating the influence of HBOT.

Previous studies have reported the beneficial influence of HBOT on anastomotic healing such as increasing ABP [22, $38,39]$ and reducing anastomotic adhesions. This was also observed in our study. Moreover, the reduction of intraabdominal abscess formation indicates an anti-infection effect of HBOT playing an important role in its overall beneficial effect. Because tissue necrosis and ischemia-reperfusion injury caused by the anastomotic ischemia also impair the systematic condition, we expected such beneficial effect of HBOT might also be observed in addition to the localized changes. Though failed to reduce the mortality rate, the HBOT group resulted in better kidney function as a marker for general health on POD 7, indicating a beneficial systematic effect. More importantly, the preconditioning effect remained after cessation of HBOT.

Perioperative oxygen therapy providing $100 \%$ oxygen under higher pressure has been demonstrated to be effective to prevent CAL, but the mechanism is not fully understood. Moreover, it also influences the vascular response. The high concentration of oxygen causes vessel constriction, which has also been reported on HBO therapy cases [40, 41]. Though not fully understood, our preliminary observation found a different response pattern in the HBOT groups after artery ligation (unpublished data), which may eventually influence the blood supply and oxygenation of the tissues after anastomosis construction.

Substantial amount of data demonstrate that the effect of HBOT is not limited to the direct oxygenation $[12,13]$. Previous studies showed that HBO therapy increased the $\mathrm{ABP}$ even when anastomoses were constructed under contaminated conditions [42, 43]. HBOT has been demonstrated to increase expression of the anti-inflammatory genes as well as influencing the local production of inflammatory cytokines [43], many of which are productions of macrophages. In 

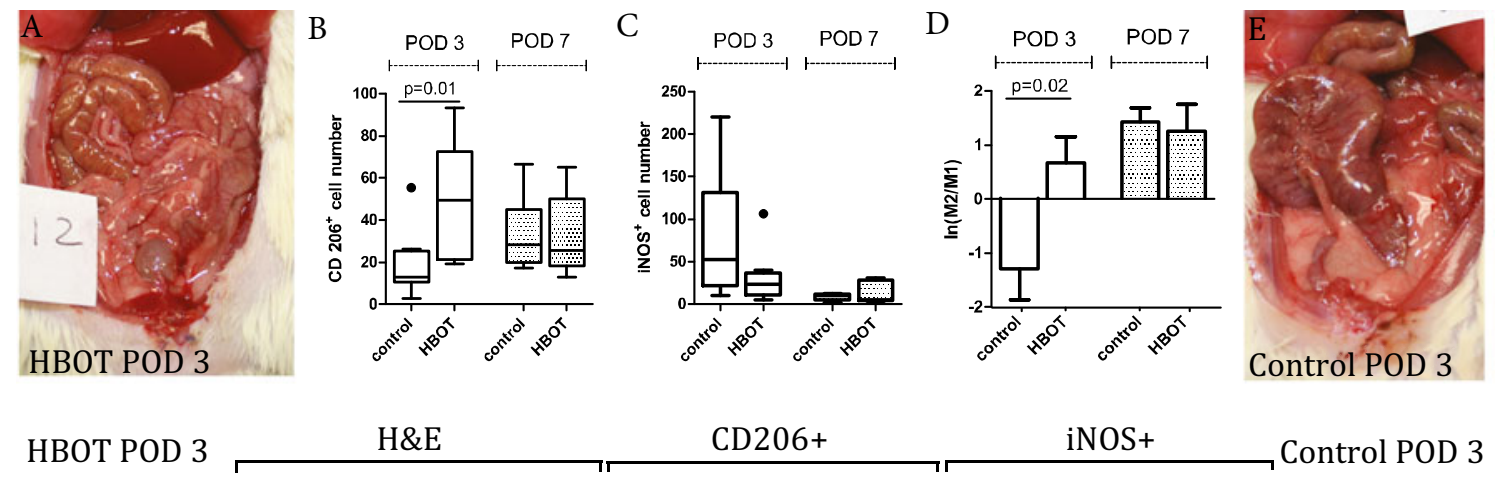

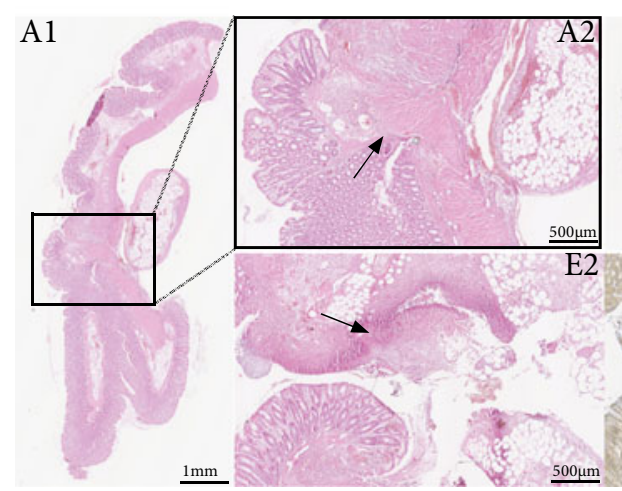

Fig. 5 Comparison of macrophage numbers at the anastomotic site on POD 3 and POD 7 of the HBOT groups and control groups. a and $\mathbf{e}$ are representative pictures of an overview of the abdomen at POD 3. a A picture of a rat from the HBOT group which has no necrosis or ischemia. $E$ shows ischemia of the cecum. b Illustrates the CD206+ cell number (indication of M2 macrophages), the dot above the first bar indicate an outlier. c Illustrates the iNOS + cell number (indication of M1 macrophages), the dot above the second bar indicate an outlier. $\mathbf{d}$ Illustrates the M2/M1 index which is significantly in advantage of the
HBOT POD3 group; $p=0.02$. The left side of the image represents an anastomosis of the HBOT POD 3 group, the left side of this image is the intraluminal side of the colon. The selected area in $A 1$ is represented in $A 2$. Of the same area the immunohistochemistry staining of CD206+ and iNOS+ is represented in $A 3$ and $A 4$, positive cells are colored with diaminobenzidine. The same is true for the representative histology slides for the control POD3 groups as shown in figure E1-E4. The arrows in $A 2$ and $E 2$ indicate the anastomotic line ( $20 \times 10$ magnification) accordance with that, our data suggest that the influence of HBOT on the inflammatory response via alternatively activating the macrophages. In addition to the increased ABP, the higher M2/M1 index also explains for a reduction of adhesion formation on anastomosis, probably because of earlier onset of regeneration as M2 macrophages enhance the regenerative responses as production of collagen [44, 45].

We investigated the mechanism of the HBOT on colorectal healing using perioperative treatment format which according to the literature has demonstrated the most beneficial effect using perioperative HBOT [39]. We are aware that it is difficult to select the patients who would have a greater risk of ischemic anastomosis and start the treatment preoperatively. However, in some patients, such disposition can be presumed preoperatively. Clinical data remain in query to investigate whether application of HBOT in high-risk patients (e.g., smokers, patients with atherosclerosis, diabetic mellitus, cardiovascular disease, and low colon perfusion during operation due to low blood pressure, blood loss or multiple organ failure) may reduce CAL rate and improve clinical outcome. Whether such application would benefit in a larger scale of patients (i.e. patients without clear risk of CAL) needs further investigation in the future.

\section{Conclusion}

Perioperative hyperbaric oxygen therapy prevents anastomotic leakage in ischemic colorectal anastomosis in the rat. The presence of anti-inflammatory macrophages is associated with the anastomotic healing. Application of HBOT as adjuvant therapy might be useful in the clinic when the patient is critically ill, and given the results of this study, this needs testing in the near future.

Compliance with ethical standards The experimental protocol was approved by the local Ethical Committee of Animal Experimentation of Erasmus University Rotterdam.

Conflict of interest The authors declare that they have no conflict of interest.

Grant support None. 
Open Access This article is distributed under the terms of the Creative Commons Attribution 4.0 International License (http:// creativecommons.org/licenses/by/4.0/), which permits unrestricted use, distribution, and reproduction in any medium, provided you give appropriate credit to the original author(s) and the source, provide a link to the Creative Commons license, and indicate if changes were made.

\section{References}

1. Komen $\mathrm{N}$ et al (2008) Anastomotic leakage, the search for a reliable biomarker. A review of the literature. Color Dis 10(2):109-115, discussion 115-7

2. Peeters $\mathrm{KC}$ et al (2005) Risk factors for anastomotic failure after total mesorectal excision of rectal cancer. Br J Surg 92(2):211-216

3. Lovegrove RE et al (2006) A comparison of hand-sewn versus stapled ileal pouch anal anastomosis (IPAA) following proctocolectomy: a meta-analysis of 4183 patients. Ann Surg 244(1):18-26

4. van Vugt JL et al (2014) Improving the outcomes in oncological colorectal surgery. World J Gastroenterol 20(35):12445-12457

5. Vignali A et al (2000) Altered microperfusion at the rectal stump is predictive for rectal anastomotic leak. Dis Colon Rectum 43(1):7682

6. Boyle NH et al (2000) Intraoperative assessment of colonic perfusion using scanning laser Doppler flowmetry during colonic resection. J Am Coll Surg 191(5):504-510

7. Sheridan WG, Lowndes RH, Young HL (1987) Tissue oxygen tension as a predictor of colonic anastomotic healing. Dis Colon Rectum 30(11):867-871

8. Kologlu M et al (2000) Effect of local and remote ischemiareperfusion injury on healing of colonic anastomoses. Surgery 128(1):99-104

9. Kingham TP, Pachter HL (2009) Colonic anastomotic leak: risk factors, diagnosis, and treatment. J Am Coll Surg 208(2):269-278

10. Law WI et al (2000) Risk factors for anastomotic leakage after low anterior resection with total mesorectal excision. Am J Surg 179(2): 92-96

11. Guo S, Dipietro LA (2010) Factors affecting wound healing. J Dent Res 89(3):219-229

12. Eisenbud DE (2012) Oxygen in wound healing: nutrient, antibiotic, signaling molecule, and therapeutic agent. Clin Plast Surg 39(3): 293-310

13. Tandara AA, Mustoe TA (2004) Oxygen in wound healing-more than a nutrient. World J Surg 28(3):294-300

14. Haglund U, Bulkley GB, Granger DN (1987) On the pathophysiology of intestinal ischemic injury. Clinical review. Acta Chir Scand 153(5-6):321-324

15. Klemetti E, Rico-Vargas S, Mojon P (2005) Short duration hyperbaric oxygen treatment effects blood flow in rats: pilot observations. Lab Anim 39(1):116-121

16. Kemik O et al (2013) Evaluation of the effects of hyperbaric oxygen treatment and enoxaparin on left colon anastomosis. An experimental study. Eur Rev Med Pharmacol Sci 17(17):2286-2292

17. Hohn DC et al (1976) Effect of O2 tension on microbicidal function of leukocytes in wounds and in vitro. Surg Forum 27(62):18-20

18. Schietroma $\mathrm{M}$ et al (2012) Colorectal Infraperitoneal anastomosis: the effects of perioperative supplemental oxygen administration on the anastomotic dehiscence. J Gastrointest Surg 16(2):427-434

19. Bickel A et al (2011) Perioperative hyperoxygenation and wound site infection following surgery for acute appendicitis: a randomized, prospective, controlled trial. Arch Surg 146(4):464-470
20. Dauwe PB et al (2014) Does hyperbaric oxygen therapy work in facilitating acute wound healing: a systematic review. Plast Reconstr Surg 133(2):208e-215e

21. Svalestad J et al (2014) Effect of hyperbaric oxygen treatment on oxygen tension and vascular capacity in irradiated skin and mucosa. Int J Oral Maxillofac Surg 43(1):107-112

22. Hamzaoglu I et al (1998) The effects of hyperbaric oxygen on normal and ischemic colon anastomoses. Am J Surg 176(5):458-461

23. Attard JA et al (2005) The effects of systemic hypoxia on colon anastomotic healing: an animal model. Dis Colon Rectum 48(7): $1460-1470$

24. Wu Z et al (2014) The prevention of colorectal anastomotic leakage with tissue adhesives in a contaminated environment is associated with the presence of anti-inflammatory macrophages. Int J Color Dis 29(12): $1507-1516$

25. Grommes $\mathrm{J}$ et al (2013) Comparison of intestinal microcirculation and wound healing in a rat model. J Invest Surg 26(1):46-52

26. Sander AL et al (2009) In vivo effect of hyperbaric oxygen on wound angiogenesis and epithelialization. Wound Repair Regen 17(2):179-184

27. Djasim UM et al (2012) A hyperbaric oxygen chamber for animal experimental purposes. Int J Oral Maxillofac Surg 41(2):271-274

28. Wu Z et al (2014) Colorectal anastomotic leakage caused by insufficient suturing after partial colectomy: a new experimental model. Surg Infect 15(6):733-738

29. Rodgers $\mathrm{K}$ et al (2000) Angiotensin II increases host resistance to peritonitis. Clin Diagn Lab Immunol 7(4):635-640

30. Verco SJ et al (2000) Development of a novel glucose polymer solution (icodextrin) for adhesion prevention: pre-clinical studies. Hum Reprod 15(8): 1764-1772

31. Zuhlke HV et al. (1990) [Pathophysiology and classification of adhesions] Pathophysiologie und Klassifikation von Adhasionen. Langenbecks Arch Chir Suppl II Verh Dtsch Ges Chir 1009-16

32. Phillips JD et al (1992) Effects of chronic corticosteroids and vitamin A on the healing of intestinal anastomoses. Am J Surg 163(1): 71-77

33. Beddy D, Dozois EJ, Pemberton JH (2011) Perioperative complications in inflammatory bowel disease. Inflamm Bowel Dis 17(7): $1610-1619$

34. Komen $\mathrm{N}$ et al (2011) High tie versus low tie in rectal surgery: comparison of anastomotic perfusion. Int J Color Dis 26(8):10751078

35. Guslandi M et al (1998) Measurement of rectal blood flow by laser Doppler flowmetry in inflammatory bowel disease. Hepatogastroenterology 45(20):445-446

36. Tateishi S, Arima S, Futami K (1997) Assessment of blood flow in the small intestine by laser Doppler flowmetry: comparison of healthy small intestine and small intestine in Crohn's disease. J Gastroenterol 32(4):457-463

37. Wilson SB, Jennings PE, Belch JJ (1992) Detection of microvascular impairment in type I diabetics by laser Doppler flowmetry. Clin Physiol 12(2):195-208

38. Erenoglu $\mathrm{C}$ et al (2003) Effect of hyperbaric oxygen on anastomoses created under the influence of 5-FU. Undersea Hyperb Med 30(4):321-326

39. Yagci $\mathrm{G}$ et al (2006) Preoperative and postoperative administration of hyperbaric oxygen improves biochemical and mechanical parameters on ischemic and normal colonic anastomoses. J Invest Surg 19(4):237-244

40. Gill AL, Bell CN (2004) Hyperbaric oxygen: its uses, mechanisms of action and outcomes. QJM 97(7):385-395

41. Tuk B et al (2014) Hyperbaric oxygen therapy to treat diabetes impaired wound healing in rats. PLoS One 9(10):e108533 
42. Sucullu I et al (2008) The effects of hyperbaric oxygen therapy on colonic anastomosis in rats with peritonitis. J Invest Surg 21(4): 195-200

43. Poyrazoglu Y et al (2015) Effects of hyperbaric oxygen and preconditioning on wound healing in colonic anastomoses. J Invest Surg 28(4):188-195
44. Vats D et al (2006) Oxidative metabolism and PGC-1beta attenuate macrophage-mediated inflammation. Cell Metab $4(1): 13-24$

45. Lucas $\mathrm{T}$ et al (2010) Differential roles of macrophages in diverse phases of skin repair. J Immunol 184(7):3964-3977 